the herbivorous zooplankton either did or did not, depending on its composition.

The conclusion is simple. The onespecies-per-trophic-level models work when the systems are indeed that simple. Real ecosystems of course contain many interconnected food chains - but when the number of chains is as small as two, the effects of nutrient enrichment depend at least in part on the structure of the food web ${ }^{9}$.

Stuart L. Pimm is in the Department of Zoology and Graduate Program in Ecology, University of Tennessee, Knoxsville,

Tennessee 39776, USA.

\section{Invisible radioactivity}

\section{W. R. Phillips}

NUCLEAR $\beta$-decay is best known as an event in which an electron is created and ejected from a nucleus at high speed. But physicists at the German heavy-ion laboratory GSI, near Darmstadt, have succeeded in observing an exceedingly rare variant in which the electron produced is born into a bound atomic orbital $^{1}$. One potential by-product of this discovery is the prospect of making an accurate measurement of the mass of the electron neutrino.

The electron created in $\beta$-decay, in which a neutron is transmuted into a proton, typically emerges from the nucleus as a freely moving particle (with a continuum of possible kinetic energies), but there is always, in principle, the possibility that it will be created in a bound, unoccupied atomic orbital of the daughter ion. Bound-state $\beta$-decay should compete best when the maximum energy available to the electron is small; but this condition also causes the lifetime of the emitter to be long. For example, ${ }^{187} \mathrm{Re}$, which $\beta$-decays to ${ }^{187} \mathrm{Os}$ with a maximum energy just under 3 kiloelectronvolts, has a radioactive half-life of $4 \times 10^{10}$ years, a truly cosmological timescale. Even in this decay, which can be used as a cosmochronometer ${ }^{2}$ to determine the age of our Galaxy, the fraction of bound-state decays may be no more than 1 per cent (ref. 3 ).

The chances of bound-state decay are considerably greater if the decaying atom is highly ionized: this leaves tightly bound inner electron shells unoccupied and available to the emerging electron, and these shells have a relatively large binding energy to be gained by the decay process. This opens up the intriguing prospect that nuclei exist that are stable in neutral atoms but which can undergo bound-state $\beta$-decay when in highly charged or bare ions (continuum decay would still be impossible). One such nucleus is ${ }^{163} \mathrm{Dy}$, which in the neutral atom is stable against $\beta$-decay by a few kiloelectronvolts, but can undergo bound-state decay into the $\mathrm{K}$ or $\mathrm{L}$ atomic shells of ${ }^{163}$ Ho when highly ionized. This presented the Darmstadt workers with an easier way to search for NATURE - VOL 360 - 26 NOVEMBER 1992 bound-state $\beta$-decay. One can start with a number of fully stripped ${ }^{163} \mathrm{Dy}$ nuclei and count the number of single-electron ${ }^{163} \mathrm{Ho}$ ions produced in a given time. All the ${ }^{163} \mathrm{Ho}$ ions must have resulted from bound-state decay, and their number is a measure of the decay probability per unit time.

The experiment was made possible by the recent construction of the heavy-ion accelerator and storage ring at Darmstadt. Up to 100 million partially ionized ${ }^{163} \mathrm{Dy}$ ions were accelerated in a heavyion synchrotron to an energy of 47.9 gigaelectronvolts, when they are moving at about two-thirds the speed of light. At this speed most Dy ions, after passing through a thin foil, have all their electrons stripped off. In this way bare ${ }^{163} \mathrm{Dy}$ ions were produced and injected into the GSI storage ring. The highly evacuated ring's magnetic elements constrained the bare nuclei to move stably around the ring orbit. Ions of the same speed and the same mass-to-charge ratio move on similar orbits, and the ring can store ions as heavy as ${ }^{63} \mathrm{Dy}$ for periods of up to a few hours without substantial loss, during which time a few could decay to give ${ }^{163}$ Ho ions.

Because these daughter ions, with their one electron, have the same massto-charge as the parent ions, they also remain in stable orbit around the ring. By passing the ion beam through a gas jet after a short accumulation time, the Darmstadt team could strip the final electron from the Hoions, which could then be extracted from the storage ring and counted. Suitably adjusted for the loss of Dy ions from the beam, the slow count rate for the appearance of Ho ions translates into a half-life for the boundstate $\beta$-decay of $47_{-4}^{+5}$ days, close to the predicted value ${ }^{4}$ of 50 days.

One interesting outcome of the experiment is the first good upper limit on the mass of the electron neutrino (betterknown experiments on the 'neutrino' mass in fact deal with the antineutrino). Bound-state $\beta$-decay (accompanied by the ejection of an antineutrino) is the inverse process of orbital electron capture, in which a nucleus reduces its charge by capturing an electron from an atomic orbital (simultaneously creating and ejecting a neutrino). A ${ }^{163} \mathrm{Ho}$ nucleus in a neutral atom decays to ${ }^{163} \mathrm{Dy}$ by capturing an electron from the atomic $M$ shell or from outer shells with less binding energy. A bare ${ }^{163}$ Dy nucleus decays into the $\mathrm{K}$ shell around a ${ }^{163} \mathrm{Ho}$ nucleus. The ratio of probabilities for the ${ }^{163}$ Dy decay and for electron capture by ${ }^{163} \mathrm{Ho}$ depends on electron densities at the nuclei and on other factors known reasonably accurately. But it also depends on the phase (or momentum) space available to the product antineutrino or neutrino which, in turn, depends on the particle's energy. This is determined by the exact mass difference between ${ }^{163} \mathrm{Dy}$ and ${ }^{163} \mathrm{Ho}$ nuclei and also by the masses of the antineutrino and neutrino, if they have mass.

Comparison of the known electroncapture rates for ${ }^{163}$ Ho decay with the decay rate measured in the GSI experiments gives independent expressions involving these separate masses. Neglecting the mass of electron antineutrino, for which the present ${ }^{5}$ upper limit is 17 electronvolts, enables limits to be placed on the mass difference between the nuclei and on the mass of the electron neutrino. The latter is less than 410 electronvolts at the 68 per cent confidence level. (There are other measures of the neutrino mass which give lower limits, but relying on another exotic radioactive process - neutrinoless double $\beta$-decay - which has yet to be observed, their result depends on the character of the neutrino. The non-observation of this process gives a mass s,7 $^{6,7}$ if the neutrino is its own antiparticle, as suggested once by Majorana. The more usual assumption, dating back to Dirac, is that the neutrino and antineutrino are distinct particles.)

Although the upper mass limit looks poor by comparison with the antineutrino mass determinations, improvements merely require better measures of the respective radioactive decay rates and of the precise mass difference between ${ }^{163}$ Dy and ${ }^{163}$ Ho nuclei. In detecting bound-state $\beta$-decay, the GSI workers have already vaulted the most formidable hurdle.

W. R. Phillips is in the Department of Physics, University of Manchester, Oxford Road, Manchester M13 9PL, UK.

\footnotetext{
1. Jung, M. et al. Phys. Rev. Lett. 69, 2164-2167 (1992). 2. Clayton, D. D. Astrophys. J. 139, 637-663 (1964). . Chen, Z., Rosenberg, L. \& Spruch, L. Phys. Rev. A35. 1981-1990 (1987)

4. Takahashi, K., Boyd, R. N., Mathews, G. J. \& Yokoi, K Phys. Rev. C36, 1522-1528 (1987)

5. Bertsch, G. F. (ed.) Review of Particle Properties, Phys Lett. B239, 1-516 (1990)

6. Witherell, M. S. Nature 331, 392-393 (1988).

7. Hykawy, J. G. et al. Phys. Rev. Lett. 67, 1708-1711 (1991).
} 